\title{
The race model inequality for censored reaction time distributions
}

\author{
STEFan Rach \\ University of Oldenburg, Oldenburg, Germany \\ and Jacobs University Bremen, Bremen, Germany \\ Adele Diederich \\ Jacobs University Bremen, Bremen, Germany \\ AND \\ Rike Steenken and Hans Colonius \\ University of Oldenburg, Oldenburg, Germany
}

\begin{abstract}
The race model inequality (RMI) introduced in Miller (1982) puts an upper limit on the amount of reaction time facilitation within the redundant-signals paradigm that is consistent with a race model. Here, it is shown through theoretical analysis and numerical simulation that inferences from the RMI test may become invalid when the experimenter misses a proportion of the responses by limiting the recording interval (right censoring) or excluding outliers from analysis (left and/or right censoring). Moreover, a correction of the inequality test for right-censored reaction time distributions is proposed.
\end{abstract}

In the redundant-signals paradigm for simple reaction time (RT), the observer must initiate a response as quickly as possible following the detection of any stimulus onset. A typical finding is a redundancy gain: Responses are faster, on average, when two or more signals are presented simultaneously than when a single signal appears. This redundant-signals effect (RSE) has often, although not always, been replicated under different experimental settings - for example, comparing uni- versus multimodal stimulation (Diederich, 1995; Diederich \& Colonius, 1987; Gielen, Schmidt, \& Van den Heuvel, 1983; Miller, 1982, 1986; Molholm, Ritter, Javitt, \& Foxe, 2004), single versus multiple stimuli within the same modality (e.g., Schwarz \& Ischebeck, 1994), or monocular versus binocular stimulation (Hughes \& Townsend, 1998; Westendorf \& Blake, 1988) - and for specific populations (see, e.g., Corballis, 1998; Miller, 2004; Reuter-Lorenz, Nozawa, Gazzaniga, \& Hughes, 1995; and Savazzi \& Marzi, 2004, all for split-brain individuals; and Marzi et al., 1996, for hemianopics).

Raab (1962) proposed a race model for simple RT, postulating that (1) each individual stimulus elicits a (normally distributed) detection process performed in parallel to the others and (2) the winner's time determines the observable RT. The race model opens up the possibility that the RSE is generated by statistical facilitation: If detection latencies are interpreted as (nonnegative) random variables, the time to detect the first of several redundant signals is faster, on average, than the detection time for any single signal. Testing the race model amounts to probing whether an observed RT speedup is too large to be attributable to statistical facilitation (viz., probability summation), no matter which distributional assumptions have been made.

A test of general race models was developed by Miller (1978, 1982), showing that

$$
\operatorname{Pr}\left(\mathrm{RT}_{X Y} \leq t\right) \leq \operatorname{Pr}\left(\mathrm{RT}_{X} \leq t\right)+\operatorname{Pr}\left(\mathrm{RT}_{Y} \leq t\right)
$$

must hold for all $t \geq 0$. This race model inequality (RMI) follows from

$$
\operatorname{Pr}_{X Y}[\min (X, Y) \leq t] \leq \operatorname{Pr}_{X}(X \leq t)+\operatorname{Pr}_{Y}(Y \leq t)
$$

for any pair of random variables $(X, Y)$ with a joint probability distribution based on $\operatorname{Pr}_{X Y}$ and with its marginal distributions identical to $\operatorname{Pr}_{X}$ and $\operatorname{Pr}_{Y}$. Thus, as was observed in Luce (1986, p. 130), the RMI test requires that the RT distributions in the single-signal conditions are identical to the corresponding (marginal) RT distributions in the redundant-signals condition (cf. Colonius, 1990). Note that, for fixed $t$, Inequality 2 corresponds to the well-known Boole's inequality (e.g., Billingsley, 1979). Neglecting possible additional components (such as motor time), the inequality stipulates that the RT distribution function for redundant stimuli is never larger than the sum of the RT distributions for the single stimuli. A violation of this inequality is interpreted as an indication of an underlying coactivation mechanism or some other strong form of nonindependence.

S. Rach, stefan.rach@uni-oldenburg.de 
Miller's $(1978,1982)$ test has become a standard tool in numerous empirical RT studies (see the references above). Moreover, it has been the subject of various theoretical and methodological studies as well (Ashby \& Townsend, 1986; Colonius, 1990, 1999; Colonius \& Ellermeier, 1997; Colonius \& Townsend, 1997; Colonius \& Vorberg, 1994; Diederich, 1992; Miller, 1986, 1991, 2004; Miller \& Ulrich, 2003; Mordkoff \& Yantis, 1991; Townsend \& Nozawa, 1995, 1997; Townsend \& Wenger, 2004; Ulrich \& Giray, 1986; Ulrich \& Miller, 1997; Ulrich, Miller, \& Schröter, 2007). For recent approaches to a KolmogorovSmirnov-type test of the inequality, see Maris and Maris (2003) and Vorberg (2008). It has been observed by several investigators that the diagnostic power of the RMI test may be compromised under certain experimental conditions. Specifically, when subjects are not discouraged from making so-called fast guesses - that is, responses given without processing the stimulus and assumed to be much faster than regular responses - or if display conditions are such that stimuli are sometimes not detected at all, it has been shown (Eriksen, 1988; Gondan \& Heckel, 2008; Miller \& Lopes, 1991; Miller \& Ulrich, 2003) that the power of the RMI test to detect violations of the race model diminishes. Here, we show that this holds not only for the case in which subjects are missing a proportion of the stimuli, but also when an experimenter excludes a proportion of the responses, by censoring the RT distributions from the left and/or the right - that is, excluding responses below and/or above a certain RT value because they are considered to be anticipations or outliers (e.g., Giray \& Ulrich, 1993; Leo, Bertini, di Pellegrino, \& Làdavas, 2008; Miller \& Van Nes, 2007; Savazzi \& Marzi, 2008; Schwarz, 2006). Furthermore, the experimenter may miss a proportion of the responses by not registering reactions given after some arbitrary upper bound; up to $8 \%$ of responses have sometimes been eliminated this way (see Gondan, Vorberg, \& Greenlee, 2007; Miller, 2007a, 2007b; Miller \& Van Nes, 2007).

\section{Race Model Inequality for Right-Censored RT Distributions}

For concreteness, in the following, we consider a simple RT experiment in which subjects have to react by pressing a button upon detecting a stimulus, where, on some of the trials, more than one stimulus is presented (redundantsignals paradigm). Let $X, Y$ denote the random latencies to process two stimuli presented in a redundant trial, with distribution functions $F_{X}, F_{Y}$, respectively. When no responses are registered $t_{\mathrm{R}}$ (in milliseconds), say, after stimulus onset, the (right-censored) distribution function for the observed RTs is

$$
\begin{aligned}
F_{X}\left(t \mid t_{\mathrm{R}}\right) & =\operatorname{Pr}\left(X \leq t \mid X \leq t_{\mathrm{R}}\right) \\
& =\operatorname{Pr}\left(\{X \leq t\} \cap\left\{X \leq t_{\mathrm{R}}\right\}\right) / \operatorname{Pr}\left(X \leq t_{\mathrm{R}}\right),
\end{aligned}
$$

by definition of conditional probability and assuming $\operatorname{Pr}\left(X \leq t_{\mathrm{R}}\right)>0$. It follows that

$$
F_{X}\left(t \mid t_{\mathrm{R}}\right)= \begin{cases}F_{X}(t) / F_{X}\left(t_{\mathrm{R}}\right), & \text { if } t \leq t_{\mathrm{R}} \\ 1, & \text { otherwise. }\end{cases}
$$

Analogous equations hold for the random variables $Y$ and $\min (X, Y)$. Thus, the RMI that is actually tested in an experiment in which no responses longer than $t_{\mathrm{R}}$ are recorded is not

$$
F_{X Y}(t) \leq F_{X}(t)+F_{Y}(t)
$$

but, rather,

$$
F_{X Y}\left(t \mid t_{\mathrm{R}}\right) \leq F_{X}\left(t \mid t_{\mathrm{R}}\right)+F_{Y}\left(t \mid t_{\mathrm{R}}\right)
$$

or, equivalently,

$$
F_{X Y}(t) / F_{X Y}\left(t_{\mathrm{R}}\right) \leq F_{X}(t) / F_{X}\left(t_{\mathrm{R}}\right)+F_{Y}(t) / F_{Y}\left(t_{\mathrm{R}}\right),
$$

writing $F_{X Y}$ for the distribution function in the redundantsignals condition. Multiplying both sides of this inequality by $F_{X Y}\left(t_{\mathrm{R}}\right)$ yields

$$
\begin{aligned}
F_{X Y}(t) \leq & F_{X}(t) \times F_{X Y}\left(t_{\mathrm{R}}\right) / F_{X}\left(t_{\mathrm{R}}\right) \\
& +F_{Y}(t) \times F_{X Y}\left(t_{\mathrm{R}}\right) / F_{Y}\left(t_{\mathrm{R}}\right) .
\end{aligned}
$$

Note that under the race model assumption, by the monotonicity of probability,

$$
\max [\operatorname{Pr}(X \leq t), \operatorname{Pr}(Y \leq t)] \leq \operatorname{Pr}(\{X \leq t\} \cup\{Y \leq t\})
$$

for all $t$. Thus, in particular,

$$
\max \left[F_{X}\left(t_{\mathrm{R}}\right), F_{Y}\left(t_{\mathrm{R}}\right)\right] \leq F_{X Y}\left(t_{\mathrm{R}}\right) .
$$

This inequality, sometimes called the Grice inequality (Grice, Canham, \& Boroughs, 1984), implies that the ratios $F_{X Y}\left(t_{\mathrm{R}}\right) / F_{X}\left(t_{\mathrm{R}}\right) \equiv r_{X}$ and $F_{X Y}\left(t_{\mathrm{R}}\right) / F_{Y}\left(t_{\mathrm{R}}\right) \equiv r_{Y}$ in Inequality 5 are larger than or equal to one. Thus, testing Inequality 4 is equivalent to testing

$$
F_{X Y}(t) \leq r_{X} \times F_{X}(t)+r_{Y} \times F_{Y}(t) .
$$

Since the right-hand side of this inequality may be larger than $F_{X}(t)+F_{Y}(t)$ for certain values of $t$, it may happen that the RMI for right-censored RT, Inequality 4 , is satisfied for all $t$, whereas the "true" (i.e., uncensored) RMI, Inequality 3 , is violated for certain values of $t$. Thus, for right-censored distributions, the RMI test becomes less diagnostic: It may indicate no violation of the race model, although, in fact, RT facilitation is stronger than predicted by the race model. In other words, if the RMI test for right-censored data indicates a violation of the race model, it is safe to assume that the redundancy gain is, in fact, large enough to suggest some type of coactivation mechanism.

Effect of right censoring due to missing trials: Numerical example. This effect of right censoring is illustrated here assuming gamma distributions (see the Appendix) for all three latencies: $X \sim \operatorname{gamma}(2,1 / 300)$, $Y \sim \operatorname{gamma}(2,1 / 250)$, and $X Y \sim \operatorname{gamma}(2,1 / 140)$, where $X Y$ denotes the redundant-signals latency. To see that this model does not satisfy the RMI, consider the function

$$
R(t)=F_{X Y}(t)-\min \left\{F_{X}(t)+F_{Y}(t), 1\right\} .
$$

Obviously, the RMI is violated whenever this function takes on positive values. The upper left panel in Figure 1 shows violations with the chosen gamma distributions when $t$ is small enough. 


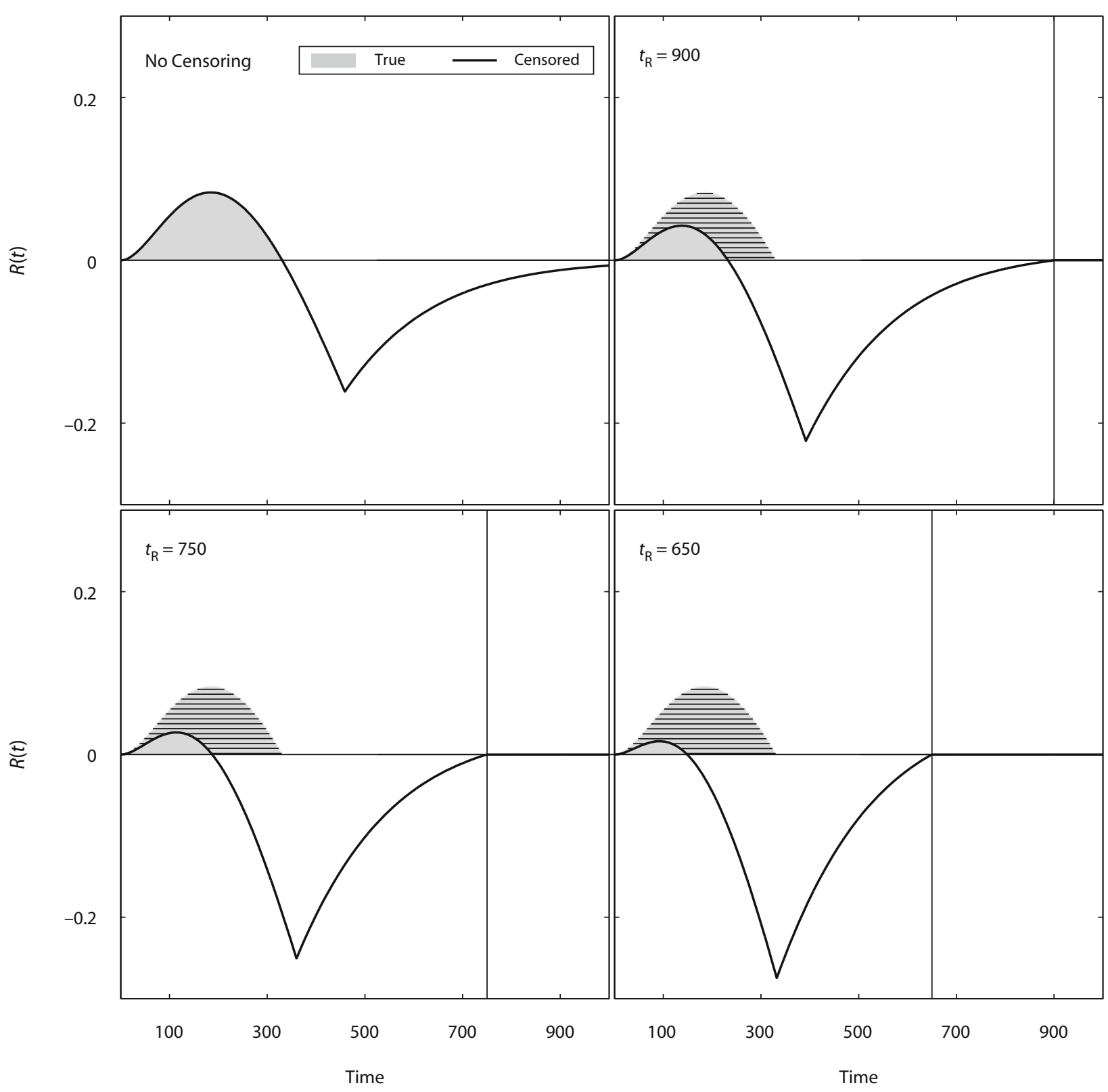

Figure 1. Positive values of the function $R(t)$ indicate violations of the race model inequality. Underlying distributions are gamma with a shape parameter of 2 and scale parameters of $1 / 300(X), 1 / 250(Y)$, and 1/140 (redundant). The gray area below $R(t)$ is a measure of the amount of violation. As censoring is increased (by shifting the vertical line indicating the cut point $t_{\mathrm{R}}$ to the left), this area shrinks (horizontal hatching indicates the amount of decrease). Different subplots present results for truncation times $t_{\mathrm{R}}=+\infty, 900$, 750 , and 650 msec.

Taking the shaded area above the abscissa defined by $R(t)$ as an index of the magnitude of violation (Colonius $\&$ Diederich, 2006; Miller, 1986), the other panels of the figure illustrate how this area shrinks to zero with decreasing $t_{\mathrm{R}}$ - that is, with amplifying censoringmaking it more and more difficult to detect any violation of the RMI.

\section{Race Model Inequality for}

\section{Left-Censored RT Distributions}

Under the same experimental setting, if responses faster than or equal to $t_{\mathrm{L}}$ (in milliseconds) are considered anticipations and, therefore, excluded from analysis, the left-censored probability distribution function for the remaining RTs is

$$
\begin{aligned}
F_{X}\left(t \mid t_{\mathrm{L}}\right) & =\operatorname{Pr}\left(X \leq t \mid X>t_{\mathrm{L}}\right) \\
& =\operatorname{Pr}\left(t_{\mathrm{L}}<X \leq t\right) /\left[1-\operatorname{Pr}\left(X \leq t_{\mathrm{L}}\right)\right],
\end{aligned}
$$

by definition of conditional probability and assuming $\operatorname{Pr}\left(X>t_{\mathrm{L}}\right)>0$. It follows that

$$
F_{X}\left(t \mid t_{\mathrm{L}}\right)= \begin{cases}{\left[F_{X}(t)-F_{X}\left(t_{\mathrm{L}}\right)\right] /\left[1-F_{X}\left(t_{\mathrm{L}}\right)\right],} & \text { if } t>t_{\mathrm{L}} \\ 0, & \text { otherwise. }\end{cases}
$$


Analogous equations hold for the random variables $Y$ and $\min (X, Y)$. Thus, the RMI actually tested in an experiment in which no responses shorter than or equal to $t_{\mathrm{L}}$ are recorded is not

$$
F_{X Y}(t) \leq F_{X}(t)+F_{Y}(t)
$$

but, rather,

$$
F_{X Y}\left(t \mid t_{\mathrm{L}}\right) \leq F_{X}\left(t \mid t_{\mathrm{L}}\right)+F_{Y}\left(t \mid t_{\mathrm{L}}\right),
$$

or equivalently, for $t>t_{\mathrm{L}}$,

$$
\frac{F_{X Y}(t)-F_{X Y}\left(t_{\mathrm{L}}\right)}{1-F_{X Y}\left(t_{\mathrm{L}}\right)} \leq \frac{F_{X}(t)-F_{X}\left(t_{\mathrm{L}}\right)}{1-F_{X}\left(t_{\mathrm{L}}\right)}+\frac{F_{Y}(t)-F_{Y}\left(t_{\mathrm{L}}\right)}{1-F_{Y}\left(t_{\mathrm{L}}\right)},
$$

assuming nonzero denominators. Multiplying both sides of this inequality by $1-F_{X Y}\left(t_{\mathrm{L}}\right)$ and adding $F_{X Y}\left(t_{\mathrm{L}}\right)$ yields

$$
\begin{aligned}
F_{X Y}(t) \leq F_{X Y}\left(t_{\mathrm{L}}\right) & +\left[F_{X}(t)-F_{X}\left(t_{\mathrm{L}}\right)\right] \cdot \frac{1-F_{X Y}\left(t_{\mathrm{L}}\right)}{1-F_{X}\left(t_{\mathrm{L}}\right)} \\
& +\left[F_{Y}(t)-F_{Y}\left(t_{\mathrm{L}}\right)\right] \cdot \frac{1-F_{X Y}\left(t_{\mathrm{L}}\right)}{1-F_{Y}\left(t_{\mathrm{L}}\right)}
\end{aligned}
$$$$
\equiv H(t)
$$

From Inequality 6 , both $\left[1-F_{X Y}\left(t_{\mathrm{L}}\right)\right] /\left[1-F_{Y}\left(t_{\mathrm{L}}\right)\right]$ and $\left[1-F_{X Y}\left(t_{\mathrm{L}}\right)\right] /\left[1-F_{X}\left(t_{\mathrm{L}}\right)\right]$ are less than or equal to one, implying

$$
\begin{aligned}
H(t) & \leq F_{X Y}\left(t_{\mathrm{L}}\right)+\left[F_{X}(t)-F_{X}\left(t_{\mathrm{L}}\right)\right]+\left[F_{Y}(t)-F_{Y}\left(t_{\mathrm{L}}\right)\right] \\
& =F_{X}(t)+F_{Y}(t)+F_{X Y}\left(t_{\mathrm{L}}\right)-\left[F_{X}\left(t_{\mathrm{L}}\right)+F_{Y}\left(t_{\mathrm{L}}\right)\right] \\
& =F_{X}(t)+F_{Y}(t)+c,
\end{aligned}
$$

where $c \equiv F_{X Y}\left(t_{\mathrm{L}}\right)-\left[F_{X}\left(t_{\mathrm{L}}\right)+F_{Y}\left(t_{\mathrm{L}}\right)\right]$ is smaller than or equal to zero if RMI holds at $t_{\mathrm{L}}$. Therefore, under the race model assumption, we have

$$
F_{X Y}(t) \leq H(t) \leq F_{X}(t)+F_{Y}(t)+c,
$$

with the first inequality representing the test performed under left censoring. Obviously, for negative $c$, the upper bound $H(t)$ constitutes a test stronger than that required by the race model when no left censoring has been done. In other words, under left censoring, the inequality test of Inequality 9 may signal violations of the race model even though it actually holds - that is, left censoring may generate false alarms.

On the other hand, it is important to realize that, at the same time, under left censoring, an experimenter using the test of Inequality 9 will also miss all potential violations of the race model occurring before the censoring time $t_{\mathrm{L}}$.

Effect of left censoring due to missing trials: $\mathrm{Nu}$ merical example. Assuming, as before, gamma distributions for all three latencies - $X \sim \operatorname{gamma}(2,1 / 300), Y \sim$ $\operatorname{gamma}(2,1 / 250)$, and $X Y \sim \operatorname{gamma}(2,1 / 140)$ — the effect of left censoring is illustrated in Figure 2.

Again, the shaded area above the abscissa defined by $R(t)$ is an index of the magnitude of violation (cf. Equation 8). The upper left panel in Figure 2 shows violations with the chosen gamma distribution when $t$ is large enough. The other panels of the figure illustrate how this area is influenced by increasing $t_{\mathrm{L}}$. With amplifying left censoring, the amount of true violations not detected increases (horizontal hatching), whereas the amount of violations detected in the censored distributions but not present in the original distributions increases (vertical hatching).

\section{Correcting for Right Censoring}

The results obtained so far clearly suggest that one should avoid censoring whenever possible (see Ulrich \& Miller, 1994). Nevertheless, there are situations in which censoring is a consequence of the experimental setup or paradigm. For instance, in simple response tasks with weak stimuli, a response is recorded as missing whenever the subject fails to respond within a certain time period (e.g., Gondan et al., 2007; Miller, 2007a, 2007b; Miller \& Van Nes, 2007), which amounts to right censoring by the end of the recording interval $t_{\mathrm{R}}$. The proportion of trials on which an experimenter fails to record an RT in a given condition because no response was given is usually an observable quantity. Let $p_{X}, p_{Y}, p_{X Y}$ denote the corresponding probabilities for single-stimulus and redundant-stimulus presentations, respectively, with $p_{X}=1-F_{X}\left(t_{\mathrm{R}}\right), p_{Y}=$ $1-F_{Y}\left(t_{\mathrm{R}}\right), p_{X Y}=1-F_{X Y}\left(t_{\mathrm{R}}\right)$. Then, ${ }^{1}$ Inequality 4 can be transformed back into the uncensored Inequality 3 by multiplying each of the distributions by the corresponding proportion,

$$
\begin{aligned}
F_{X Y}\left(t \mid t_{\mathrm{R}}\right) \times\left(1-p_{X Y}\right) \leq & F_{X}\left(t \mid t_{\mathrm{R}}\right) \times\left(1-p_{X}\right) \\
& +F_{Y}\left(t \mid t_{\mathrm{R}}\right) \times\left(1-p_{Y}\right) .
\end{aligned}
$$

In practice, when only estimates of the proportions of missing reactions (and of all distribution functions) are available, the inequality above will only approximate the "true" inequality (3). An implementation of this correction is straightforward. First, choose the replacement time $t_{\max }$ to equal the largest RT in the whole data set increased by 1 . Second, for every missing trial, set the corresponding RT to $t_{\max }$. The corrected data set can then be subjected to common algorithms for the RMI test without further modifications (e.g., Ulrich et al., 2007). An analogous correction formula for simultaneous left and right censoring is given in the Appendix.

\section{Amount of Inferential Errors \\ Caused by Censoring: Simulation Results}

As is shown above, censoring may lead to two different kinds of inferential error: (1) A violation may occur in the original data but not in the censored data (i.e., a miss), or (2) no violation occurs in the original data but one is indicated in the censored data (i.e., a false alarm).

To investigate the amount of inferential errors that may be caused by censoring, a simulation study for simple RT experiments was conducted. RTs were assumed to be Weibull distributed for both single- and redundant-target conditions. The Weibull distribution (see the Appendix) has previously been used to describe RT distributions in a variety of situations (see Colonius, 1995; Hsu, 2005; Logan, 1992; Maloney \& Wandell, 1984; Marley, 1989; Marley \& Colonius, 1992; McGill, 1963). An additional constant base time of $200 \mathrm{msec}$ was assumed. The shape 


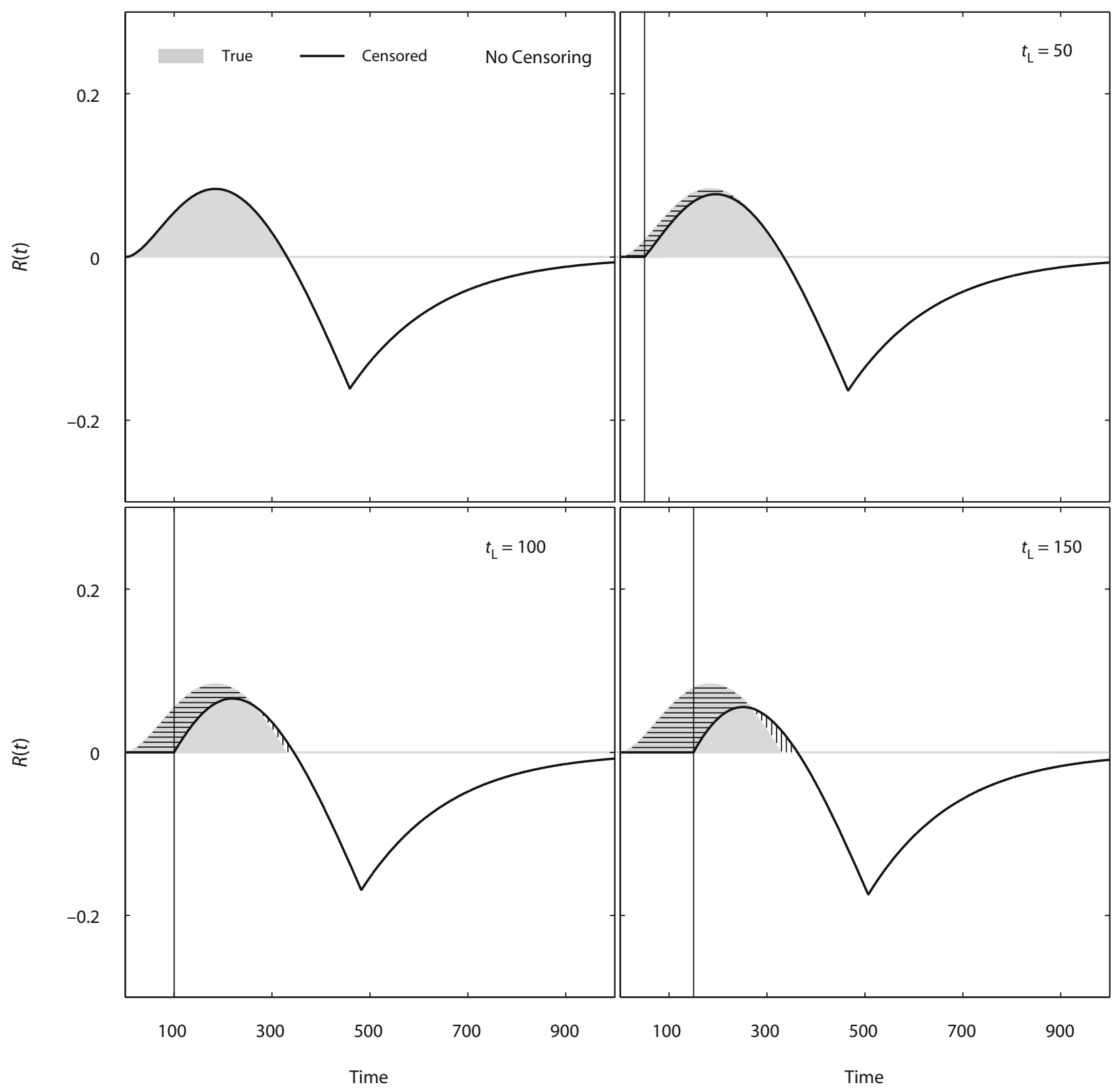

Figure 2. Positive values of the function $R(t)$ indicate violations of the race model inequality. Underlying distributions are gamma with a shape parameter of 2 and scale parameters of $1 / 300(X), 1 / 250(Y)$, and 1/140 (redundant). The gray area below $R(t)$ is a measure of the amount of violation. As censoring is increased (by shifting the vertical line indicating the cut point $t_{\mathrm{L}}$ to the right), the amount of true violations not detected increases for the censored distributions (horizontal hatching), whereas the amount of violations detected in the censored distributions but not present in the original distributions increases (vertical hatching). Different subplots present results for truncation times $t_{\mathrm{L}}=0,50,100$, and 150 msec.

parameter of the Weibull was set to $\alpha=1.5$ for all conditions. The scale parameters for the distributions of the single-target conditions, $X$ and $Y$, were set to $\lambda_{X}=\lambda_{Y}=$ 0.01 . To study the influence of the magnitude of coactivation gain (i.e., the RT gain exceeding the race model prediction), three different scale parameters were assumed for the redundant-target condition, $Z$. The scale parameter was varied as follows: no coactivation gain - that is, a race model $\left(\right.$ gain $=0 \mathrm{msec} ; \lambda_{Z}=\left(\lambda_{X}^{\alpha}+\lambda_{Y}^{\alpha}\right)^{1 / \alpha}=$ 0.0159 ) — a small coactivation gain (gain $=5 \mathrm{msec} ; \lambda_{Z}=$ 0.0174 ), and a large coactivation gain (gain $=10 \mathrm{msec}$;
$\left.\lambda_{Z}=0.0193\right)$. The effect of the level of censoring was studied by excluding $1 \%, 2.5 \%, 5 \%, 7.5 \%, 10 \%$, or $15 \%$ of the original data set. Furthermore, the direction of censoring was varied by excluding data from the left, from the right, or from both directions, ${ }^{2}$ resulting in a total of 18 censoring conditions ( 6 levels $\times 3$ directions). To study the appropriateness of the proposed correction procedures, the uncensored distributions were reconstructed using Inequality 12 for right-censored data, Inequality A2 with $t_{\mathrm{R}}=\infty$ for left-censored data, and Inequality A2 for data censored from both directions. 
Under these conditions, 10,000 simple RT experiments were simulated as follows. For each of 20 (virtual) subjects, samples of 40 RTs were drawn from each of $X, Y$, and $Z$. From these samples, the cumulative distribution functions (CDFs) $F_{X}, F_{Y}$, and $F_{Z}$ were estimated. ${ }^{3}$ Furthermore, the right-hand side of RMI, $F_{S}(t)$, was computed as follows:

$$
F_{S}(t)= \begin{cases}F_{X}(t)+F_{Y}(t), & F_{X}(t)+F_{Y}(t) \leq 1 \\ 1, & \text { otherwise. }\end{cases}
$$

From $F_{Z}$ and $F_{S}$, the percentiles $\left(\hat{z}_{0.05}, \ldots, \hat{z}_{0.5}\right)$, and $\left(\hat{s}_{0.05}, \ldots, \hat{s}_{0.5}\right)$ were computed. Testing the RMI for percentiles above the $50 \%$ percentile seems not sensible, because violations against the RMI are harder to detect as $F_{X}(t)+F_{Y}(t)$ becomes large relative to $F_{Z}(t)$ (Miller, 1982). For each of the percentiles, paired $t$ tests were computed across all subjects, with $\alpha$ adjusted to the number of percentiles tested (Bonferroni correction). ${ }^{4}$ Then, for each of the 18 censoring conditions, the original samples drawn from $X, Y$, and $Z$ were censored, and the resulting samples were subjected to the same testing procedure as the original samples (described above). Finally, the CDFs from the censored samples were corrected using the formulas in Inequality 12 and Inequality A2, and the resulting data were also subjected to the testing procedure. For each of these 10,000 simulated experiments, it was thus determined whether or not a violation occurred in the original, the censored, or the corrected sample.

The results of the simulation are given in Figure 3, presenting the influence of censoring on the diagnosticity of the RMI test as a function of the coactivation gain (in milliseconds), censoring method, and level of censoring. Columns present data for the different censoring cases (from left to right: censoring from the left, censoring from the right, and censoring from both sides simultaneously). Rows present simulations for different coactivation gains (from top to bottom: 0,5 , and $10 \mathrm{msec}$ ). In each plot, the horizontal black lines indicate the total number of violations of the RMI detected in the original data in 10,000 simulated experiments (i.e., the "true" number of violations). Black (respectively, gray) bars indicate the number of violations detected in the censored (respectively, corrected) data when a violation was detected in the original data for the same experiment (i.e., a hit). Black (or gray) bars smaller than the black horizontal line indicate conditions in which the RMI is less sensitive for the respective samples - that is, where violations are detected in the original sample, but none are detected in the censored (respectively, corrected) sample. White bars stacked on top of the black and gray bars indicate the number of false alarms - that is, experiments in which a violation was detected in the modified data, but none was detected in the original data. For the no coactivation gain condition (upper left panel), for instance, violations against the RMI were detected for 108 out of 10,000 simulated experiments (represented by the horizontal line). When $1 \%$ of the data were discarded by left censoring, violations were detected for only 51 out of these 108 experiments (leftmost black bar). In addition, violations were detected for 67 experiments for which no violation was indicated in the original data (i.e., false alarms; white bar stacked on top of the leftmost black bar). The black and the white bars together cutting across the horizontal line indicates that left censoring led to an RMI test stronger than that required by the race model when no left censoring has been done. When the censored data were corrected, 107 out of 108 "true" violations were detected (leftmost gray bar) and, in addition, 22 false alarms occurred (white bar stacked on top of the leftmost gray bar). The RMI test for the corrected data is also stronger than required by the race model for the original data, since the gray and the white bars together cut across the horizontal line.

Figure 3 clearly indicates that censoring can dramatically decrease the diagnosticity of the RMI, since the number of hits lies systematically below the "true" number of violations for almost all the censored conditions (see the black bars), and the situation worsens with increasing proportions of excluded RTs. The rightcensoring results replicate the previous findings with the gamma distribution. Furthermore, for left censoring and censoring from both directions (leftmost and rightmost columns), the number of false alarms increases with increasing proportions of excluded RTs, whereas no false alarms occur for right-censored data (middle column). The gray bars indicate that the suggested correction for right-censored data is capable of restoring the diagnosticity of the RMI. However, for left censoring and censoring from both directions, the correction is not capable of countermanding the errors due to censoring, since, for most of the conditions in the leftmost and rightmost columns, the gray bars fall below the horizontal lines and false alarms occur very frequently.

Results similar to the Weibull and the gamma distribution example studied here should occur with many other distribution families. In particular, we would expect the effect of censoring to become more severe when RT distributions are heavy-tailed, like the log-normal or the Pareto distribution.

\section{Concluding Remarks}

We have studied the effect of censoring RT distributions on the performance of the RMI test. Theoretical analysis and numerical simulations revealed that the performance of the RMI test may be severely compromised under both left and right censoring. In particular, simulation results indicate that even moderate censoring of less than $5 \%$ of the total data set may strongly inflate the number of RMI violations not detected (misses) or may cause false alarms when the race model is, in fact, valid. Our recommendation, therefore, is that censoring should be avoided whenever possible.

Our analysis was based on the assumption that the rationale for censoring the distributions is somewhat pragmatic: Right-censored distributions come about because the missing trials are, indeed, very late responses occurring after the end of the recording interval. One might substantiate this assumption, for example, by applying an experimental design that involves more than one stimulus 


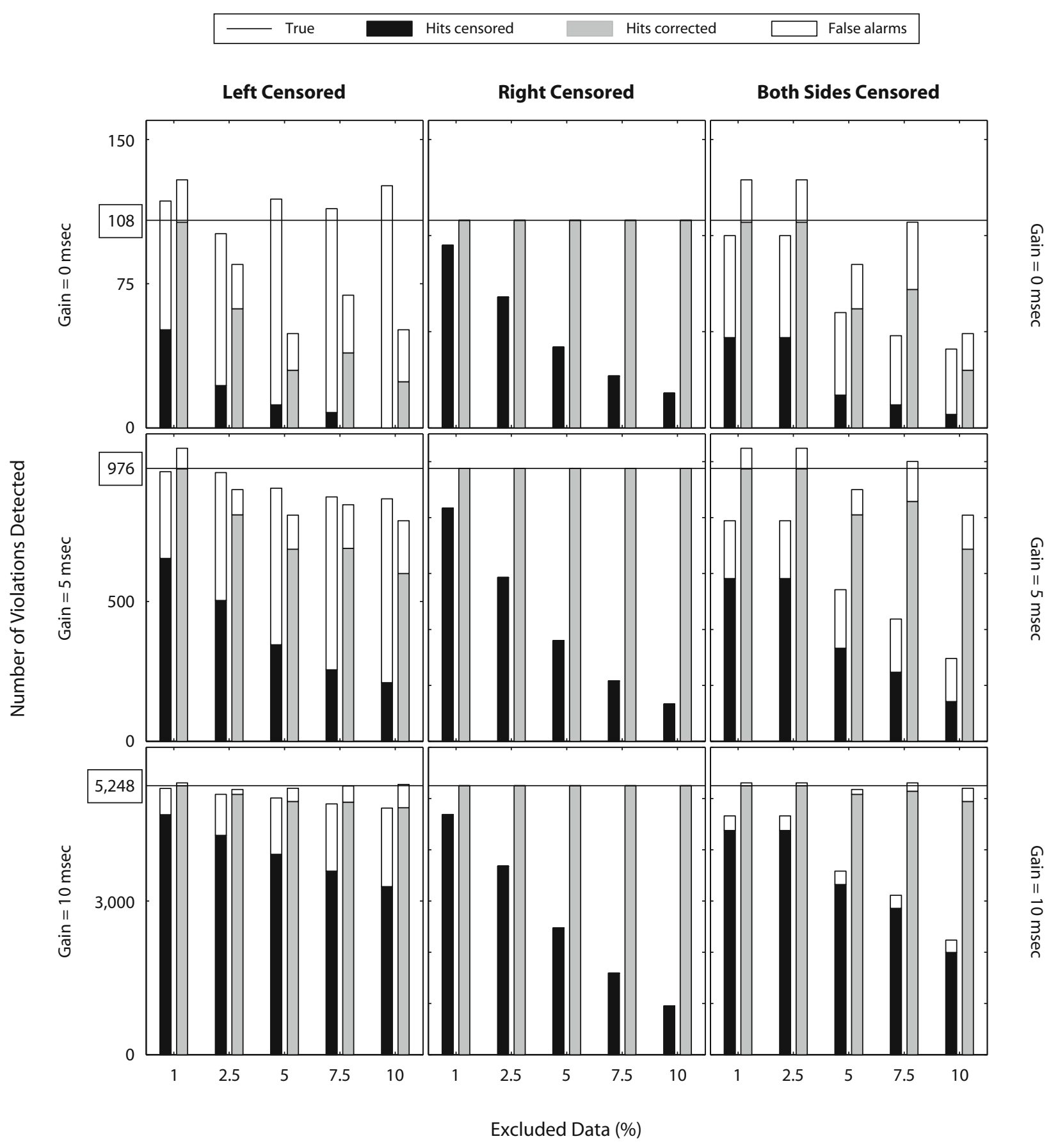

Figure 3. Influence of censoring on the diagnosticity of the race model inequality (RMI) as a function of coactivation gain (in milliseconds), censoring method, and percentage of excluded reaction times (RTs). Columns present data for different censoring methods (from left to right: censoring from the left, censoring from the right, and censoring from both sides simultaneously). Rows present simulations for different coactivation gains (i.e., the RT gain exceeding the race model prediction; from top to bottom: 0,5 , and $10 \mathrm{msec}$ ). In each plot, the horizontal black lines indicate the total number of violations against the RMI detected in the original data in 10,000 simulated experiments. Black (gray) bars indicate the number of violations detected in the censored (corrected) data when a violation was detected in the original data for the same experiment. White bars stacked on top of the black and gray bars indicate the number of false alarms - that is, experiments in which a violation was detected in the modified data but none was detected in the original data. See the text for further details.

intensity, in order to allow for the affirmation of an inverse relation between stimulus intensity and the percentage of missing trials. On the other hand, if one has reasons to assume that the observed RT distributions are actually a probability mixture resulting from different underlying processes, such as fast guesses or lapses of attention, our recommendation of avoiding censoring or for correcting the censored distributions may no longer be valid. 


\section{AUTHOR NOTE}

This research was supported by grants from the Deutsche Forschungsgemeinschaft: SFB/TR 31 Active Listening Project B4 (H.C.) and Di 506/8-1 and /-3 (A.D. and H.C.). We are most grateful to Dirk Vorberg, Toby Mordkoff, and an anonymous reviewer for very helpful comments. Correspondence concerning this article should be addressed to S. Rach, Department of Psychology, University of Oldenburg, D-26111 Oldenburg, Germany (e-mail: stefan.rach@uni-oldenburg.de).

\section{REFERENCES}

Ashby, F. G., \& Townsend, J. T. (1986). Varieties of perceptual independence. Psychological Review, 93, 154-179.

Billingsley, P. (1979). Probability and measure. New York: Wiley.

CARDILlo, G. (2006). Student t-test for unpaired or paired samples. Available at www.mathworks.com/matlabcentral/fileexchange/12699.

Colonius, H. (1990). Possibly dependent probability summation. Journal of Mathematical Psychology, 34, 253-275.

Colonius, H. (1995). The instance theory of automaticity: Why the Weibull? Psychological Review, 102, 744-750.

Colonius, H. (1999). A theorem on parallel processing models with a generalized stopping rule. Mathematical Social Sciences, 38, 247-258.

Colonius, H., \& Diederich, A. (2006). Race model inequality: Interpreting a geometric measure of the amount of violation. Psychological Review, 113, 148-154.

Colonius, H., \& Ellermeier, W. (1997). Distribution inequalities for parallel models of reaction time with an application to auditory profile analysis. Journal of Mathematical Psychology, 41, 19-27.

Colonius, H., \& Townsend, J. T. (1997). Activation-state representation of models for the redundant signals effect. In A. A. J. Marley (Ed.), Choice, decision, and measurement: Essays in honor of R. Duncan Luce (pp. 245-254). Mahwah, NJ: Erlbaum.

Colonius, H., \& Vorberg, D. (1994). Distribution inequalities for parallel models with unlimited capacity. Journal of Mathematical Psychology, 38, 35-58.

Corballis, M. C. (1998). Interhemispheric neural summation in the absence of the corpus callosum. Brain, 121, 1795-1807.

Diederich, A. (1992). Probability inequalities for testing separate activation models of divided attention. Perception \& Psychophysics, 52, 714-716.

DIEDERICH, A. (1995). Intersensory facilitation of reaction time: Evaluation of counter and diffusion coactivation models. Journal of Mathematical Psychology, 39, 197-215.

Diederich, A., \& Colonius, H. (1987). Intersensory facilitation in the motor component? A reaction time analysis. Psychological Research, 49, 23-29.

EriKsen, C. W. (1988). A source of error in attempts to distinguish coactivation from separate activation in the perception of redundant targets. Perception \& Psychophysics, 44, 191-193.

Gielen, S. C. A. M., Schmidt, R. A., \& Van den Heuvel, P. J. M. (1983). On the nature of intersensory facilitation of reaction time. Perception \& Psychophysics, 34, 161-168.

GIRAY, M., \& UlRICH, R. (1993). Motor coactivation revealed by response force in divided and focused attention. Journal of Experimental Psychology: Human Perception \& Performance, 19, 1278-1291.

Gondan, M., \& Heckel, A. (2008). Testing the race inequality: A simple correction procedure for fast guesses. Journal of Mathematical Psychology, 52, 322-325.

Gondan, M., VorberG, D., \& Greenlee, M. W. (2007). Modality shift effects mimic multisensory interactions: An event-related potential study. Experimental Brain Research, 182, 199-214.

Grice, G. R., Canham, L., \& Boroughs, J. M. (1984). Combination rule for redundant information in reaction time tasks with divided attention. Perception \& Psychophysics, 35, 451-463.

Hsu, Y.-F. (2005). On measuring the minimum detection time: A simple reaction time study in the time estimation paradigm. British Journal of Mathematical \& Statistical Psychology, 58, 259-284.

Hughes, H. C., \& Townsend, J. T. (1998). Varieties of binocular interactions in human vision. Psychological Science, 9, 53-60.

Leo, F., Bertini, C., di Pellegrino, G., \& Làdavas, E. (2008). Multisensory integration for orienting responses in humans requires the activation of the superior colliculus. Experimental Brain Research, 186, 67-77.

LoGAN, G. (1992). Shapes of reaction-time distributions and shapes of learning curves: A test of the instance theory of automaticity. Journal of Experimental Psychology: Learning, Memory, \& Cognition, 102, 883-914.

LuCE, R. D. (1986). Response times: Their role in inferring elementary mental organisation (Oxford Psychology Series, no. 8). New York: Oxford University Press.

Maloney, L. T., \& Wandell, B. A. (1984). A model of a single visual channel's response to weak test lights. Vision Research, 24, 633-640.

MARIS, G., \& MARIS, E. (2003). Testing the race model inequality: A nonparametric approach. Journal of Mathematical Psychology, 47, 507-514.

Marley, A. A. J. (1989). A random utility family that includes many of the classical models and has closed form choice probabilities and choice reaction times. British Journal of Mathematical \& Statistical Psychology, 42, 13-36.

Marley, A. A. J., \& Colonius, H. (1992). The horse race random utility model for choice probabilities and reaction times, and its competing risks interpretation. Journal of Mathematical Psychology, 36, 1-20.

Marzi, C. A., Smania, N., Martini, M. C., Gambina, G., Tomerelli, G., Palamara, A., ET Al. (1996). Implicit-redundant target effects in visual extinction. Neuropsychologia, 34, 9-22.

McGilL, W. J. (1963). Stochastic latency mechanisms. In R. D. Luce, R. R. Bush, \& E. Galanter (Eds.), Handbook of mathematical psychology (Vol. 1, pp. 309-360). New York: Wiley.

MilLER, J. (1978). Multidimensional same-different judgments: Evidence against independent comparisons of dimensions. Journal of Experimental Psychology: Human Perception \& Performance, 4, 411-422.

Miller, J. (1982). Divided attention: Evidence for coactivation with redundant signals. Cognitive Psychology, 14, 247-279.

MilleR, J. (1986). Timecourse of coactivation in bimodal divided attention. Perception \& Psychophysics, 40, 331-343.

Miller, J. (1991). Channel interaction and the redundant-targets effect in bimodal divided attention. Journal of Experimental Psychology: Human Perception \& Performance, 17, 160-169.

MilLeR, J. (2004). Exaggerated redundancy gain in the split brain: A hemispheric coactivation account. Cognitive Psychology, 49, 118154.

Miller, J. (2007a). Contralateral and ipsilateral motor activation in visual simple reaction time: A test of the hemispheric coactivation model. Experimental Brain Research, 176, 539-558.

MiLlER, J. (2007b). Interhemispheric interactions and redundancy gain: Tests of an interhemispheric inhibition hypothesis. Experimental Brain Research, 180, 389-413.

Miller, J., \& Lopes, A. (1991). Bias produced by fast guessing in distribution-based tests of race models. Perception \& Psychophysics, 50, $584-590$.

Miller, J., \& UlRich, R. (2003). Simple reaction time and statistical facilitation: A parallel grains model. Cognitive Psychology, 46, 101-151.

Miller, J., \& VAN NES, F. (2007). Effects of response task and accessory stimuli on redundancy gain: Tests of the hemispheric coactivation model. Journal of Experimental Psychology: Human Perception \& Performance, 33, 829-844.

Molholm, S., Ritter, W., Javitt, D., \& Foxe, J. J. (2004). Multisensory visual-auditory object recognition in humans: A high-density electrical mapping study. Cerebral Cortex, 14, 452-465.

Mordkoff, J. T., \& YAnTIS, S. (1991). An interactive race model of divided attention. Journal of Experimental Psychology: Human Perception \& Performance, 17, 520-538.

RAAB, D. H. (1962). Statistical facilitation of simple reaction times. Transactions of the New York Academy of Sciences, 24, 574-590.

Reuter-Lorenz, P. A., Nozawa, G., Gazzaniga, M. S., \& Hughes, H. C. (1995). Fate of neglected targets: A chronometric analysis of redundant target effects in the bisected brain. Journal of Experimental Psychology: Human Perception \& Performance, 21, 211-230.

SavazzI, S., \& MARZI, C. A. (2004). The superior colliculus subserves interhemispheric neural summation in both normals and patients with a total section or agenesis of the corpus callosum. Neuropsychologia, 42, 1608-1618. 
SAVAZZI, S., \& MARZI, C. A. (2008). Does the redundant signal effect occur at an early visual stage? Experimental Brain Research, 184, 275-281.

ScHWARz, W. (2006). On the relationship between the redundant signals effect and temporal order judgments: Parametric data and a new model. Journal of Experimental Psychology: Human Perception \& Performance, 32, 558-573.

Schwarz, W., \& Ischebeck, A. (1994). Coactivation and statistical facilitation in the detection of lines. Perception, 23, 157-168.

Shaw, M. L. (1982). Attending to multiple sources of information: I. The integration of information in decision making. Cognitive Psychology, 14, 353-409.

Townsend, J. T., \& Nozawa, G. (1995). Spatio-temporal properties of elementary perception: An investigation of parallel, serial, and coactive theories. Journal of Mathematical Psychology, 39, 321-359.

Townsend, J. T., \& Nozawa, G. (1997). Serial exhaustive models can violate the race model inequality: Implications for architecture and capacity. Psychological Review, 104, 595-602.

Townsend, J. T., \& Wenger, M. J. (2004). A theory of interactive parallel processing: New capacity measures and predictions for a response time inequality series. Psychological Review, 111, 1003-1035.

Ulrich, R., \& Giray, M. (1986). Separate-activation models with variable base time: Testability and checking of cross-channel dependency. Perception \& Psychophysics, 39, 248-254.

Ulrich, R., \& Miller, J. (1994). Effects of truncation on reaction time analysis. Journal of Experimental Psychology: General, 123, 34-80.

UlRICH, R., \& Miller, J. (1997). Tests of race models for reaction time experiments with asynchronous redundant signals. Journal of Mathematical Psychology, 41, 367-381.

Ulrich, R., Miller, J., \& Schröter, H. (2007). Testing the race model inequality: An algorithm and computer programs. Behavior Research Methods, 39, 291-302.

VORBERG, D. (2008, September). Exact statistical tests of the race-model and related inequalities. Paper presented at the 39th Meeting of the European Mathematical Psychology Group, University of Graz, Austria.

Westendorf, D. H., \& Blake, R. (1988). Binocular reaction times to contrast increments. Vision Research, 28, 355-359.

\section{NOTES}

1. In order to check for independence of these miss rates, the different tests suggested in Shaw (1982, pp. 367-369) may be considered.

2 . Excluding $x \%$ of the data by censoring from both directions was done by censoring $(x / 2) \%$ from the left and $(x / 2) \%$ from the right.

3. CDFs and percentiles were estimated using the MATLAB routine provided by Ulrich et al. (2007).

4. Paired $t$ tests were computed using the MATLAB routine provided by Cardillo (2006).

\section{APPENDIX}

\section{Gamma and Weibull Distributions}

Random variable $X$ is distributed as gamma $(\alpha, \lambda)$ if its probability density is $f(x)=\lambda \exp [-\lambda x](\lambda x)^{\alpha-1 / \Gamma(\alpha)}$ for $x>0$ and $\alpha, \lambda>0$.

Random variable $X$ is distributed as $\operatorname{Weibull}(\alpha, \lambda)$ if its (cumulative) distribution function is $F(x)=1-$ $\exp \left[-(\lambda x)^{\alpha}\right]$ for $x>0$ and $\alpha, \lambda>0$. In both cases, $\alpha$ is referred to as a shape parameter and $\lambda$ as a scale parameter.

\section{Correcting the RMI for Left and Right Censoring}

Let $t_{\mathrm{L}}<t_{\mathrm{R}}$ be the cutoffs where the RT distribution is censored from the left and right, respectively. The (leftand right-censored) distribution function for the observed RTs is

$$
\begin{aligned}
F_{X}\left(t \mid t_{\mathrm{L}}, t_{\mathrm{R}}\right) & =\operatorname{Pr}\left(X \leq t \mid t_{\mathrm{L}} \leq X \leq t_{\mathrm{R}}\right) \\
& =\operatorname{Pr}\left(t_{\mathrm{L}} \leq X \leq t\right) /\left[\operatorname{Pr}\left(X \leq t_{\mathrm{R}}\right)-\operatorname{Pr}\left(X \leq t_{\mathrm{L}}\right)\right] .
\end{aligned}
$$

The cumulative distribution function of the censored distribution is then given by

$$
F_{X}\left(t \mid t_{\mathrm{L}}, t_{\mathrm{R}}\right)= \begin{cases}0, & t<t_{\mathrm{L}} ; \\ {\left[F_{X}(t)-F_{X}\left(t_{\mathrm{L}}\right)\right] /\left[F_{X}\left(t_{\mathrm{R}}\right)-F_{X}\left(t_{\mathrm{L}}\right)\right],} & \text { for } t_{\mathrm{L}} \leq t \leq t_{\mathrm{R}} \\ 1, & \text { otherwise. }\end{cases}
$$

With $p_{X, \mathrm{~L}}=F_{X}\left(t_{\mathrm{L}}\right), p_{Y, \mathrm{~L}}=F_{Y}\left(t_{\mathrm{L}}\right), p_{X Y, \mathrm{~L}}=F_{X Y}\left(t_{\mathrm{L}}\right)$ for the proportion of left-censored data, as well as $p_{X, \mathrm{R}}=1-F_{X}\left(t_{\mathrm{R}}\right), p_{Y, \mathrm{R}}=1-F_{Y}\left(t_{\mathrm{R}}\right), p_{X Y, \mathrm{R}}=1-F_{X Y}\left(t_{\mathrm{R}}\right)$ for the proportion of right-censored data, Inequality A1 can be transformed back to Inequality 3 by

$$
\begin{aligned}
F_{X Y}\left(t \mid t_{\mathrm{L}}, t_{\mathrm{R}}\right) \times\left(1-p_{X Y, \mathrm{R}}-p_{X Y, \mathrm{~L}}\right)+p_{X Y, \mathrm{~L}} \leq & F_{X}\left(t \mid t_{\mathrm{L}}, t_{\mathrm{R}}\right) \times\left(1-p_{X, \mathrm{R}}-p_{X, \mathrm{~L}}\right) \\
& +p_{X, \mathrm{~L}}+F_{Y}\left(t \mid t_{\mathrm{L}}, t_{\mathrm{R}}\right) \times\left(1-p_{Y, \mathrm{R}}-p_{Y, \mathrm{~L}}\right)+p_{Y, \mathrm{~L}} .
\end{aligned}
$$

Note that Inequality A2 simplifies to Inequality 12 for $t_{\mathrm{L}}=0$. Setting $t_{\mathrm{R}}=\infty$ leads to a correction for leftcensored data. 